SUBJECT AREAS:

CHARACTERIZATION AND ANALYTICAL

TECHNIQUES

OILS

Received

12 February 2014

Accepted

12 May 2014

Published

29 May 2014

Correspondence and requests for materials should be addressed to M.A.A. (m.alahmad@ vaeu.ac.ae)

\title{
RF Microalgal lipid content characterization
}

\author{
Mahmoud Al Ahmad', Sulaiman Al-Zuhair ${ }^{2}$, Hanifa Taher $^{2}$ \& Ali Hilal-Alnaqbi ${ }^{3}$
}

\begin{abstract}
'Electrical Engineering Department/College of Engineering, United Arab Emirates University, P.O. Box 15551, Al-Ain, UAE, Tel: (971-3) 713 5150, Fax: (971-3) 713 4970, ${ }^{2}$ Chemical Engineering Department/College of Engineering, United Arab Emirates University, P.O. Box 15551, Al-Ain, UAE, ${ }^{3}$ Mechanical Engineering Department/College of Engineering, United Arab Emirates University, P.O. Box 15551, Al-Ain, UAE.
\end{abstract}

Most conventional techniques for the determination of microalgae lipid content are time consuming and in most cases are indirect and require excessive sample preparations. This work presents a new technique that utilizes radio frequency (RF) for rapid lipid quantification, without the need for sample preparation. Tests showed that a shift in the resonance frequency of a RF open-ended coaxial resonator and a gradual increase in its resonance magnitude may occur as the lipids content of microalgae cells increases. These response parameters can be then calibrated against actual cellular lipid contents and used for rapid determination of the cellular lipids. The average duration of lipid quantification using the proposed technique was of about 1 minute, which is significantly less than all other conventional techniques, and was achieved without the need for any time consuming treatment steps.

iodiesel produced from microalgal lipids is considered a promising alternative to fossil fuel ${ }^{1-3}$. For an economical biodiesel production from microalgae, high biomass productivity and lipid content are required. These two factors are difficult to achieve simultaneously, as conditions favoring high biomass productivity usually result in low lipid accumulation, and vice versa ${ }^{4-5}$. Under stress conditions, wherein growth rate drops, microalgae may accumulate larger lipid content ${ }^{1,6}$. This lipid accumulation should be monitored effectively for economical microalgae-biodiesel production. Therefore, a reliable and rapid quantification of lipid content is required. Although several conventional methods have been developed to determine the lipid content of microalgae cells ${ }^{7-10}$, they are in general invasive, indirect, require time treatment steps and sometimes experimentally unrepeatable. Depending on the processing steps required, it usually takes from 2 hours to 2 days to determine the amount of lipid content ${ }^{11}$. On top of that, these methods are inherently not real-time, therefore, would be very difficult to use in controlling and rapidly adjusting the growing conditions to achieve a desired objective. Therefore, it is desired to have a rapid feedback based monitoring system, which could also be integrated in-situ in a control loop, to adjust the growing conditions without the need for any time consuming and expensive sample treatment.

Electrical characterizations using mainly radio frequency (RF) techniques have received increasing attentions in the last decades due to their noninvasive characterization ability of unlimited types of materials without the need for any sample treatments ${ }^{12}$. It has been reported that RF fields that penetrate through microalgae cells are affected by the type and composition of the cells ${ }^{13}$. The RF fields propagated through microalgae suspension are altered in magnitude and phase depending on the cells lipid contents and cells concentration. This paper focuses on microalgae lipid content determination using RF, which is based on the cells intrinsic material properties that can be modeled as smart dielectric materials. Such a technique provides an accurate lipid determination in about 1 minute, which is significantly faster than the fastest lipid determining technique. A relationship is developed to correlate between the scattering parameters $\left(\mathrm{S}_{11}\right)$ and the actual lipids content. This relationship was validated by comparing its predictions against the actual values.

\section{Results}

The presented method is based on measuring high frequency scattering parameters $\left(\mathrm{S}_{11}\right)$ of microalgae suspensions placed in an open-ended coaxial RF resonator structure. The microalgae suspension affects the field distribution inside the resonator and consequently the input impedance and resonance frequencies are changed. The amount of the change depends on the intrinsic physical properties of the microalgae, namely its dielectric constant and electrical resistive losses. Since the size of the microalgae used in this work, namely Scenedesmus sp., is small, approximately in the range of several of micrometers (Fig. 1(a)), the propagation of RF field polarizes it 
(a)

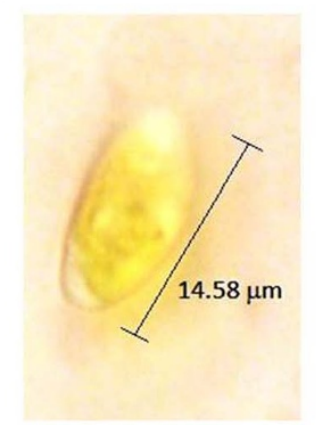

(b)

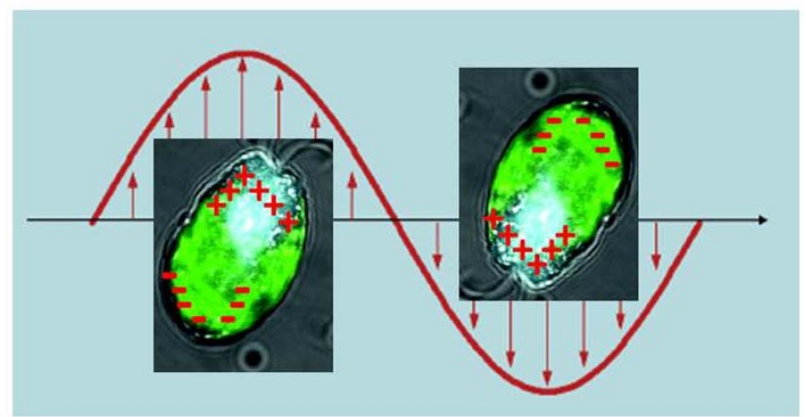

Figure $1 \mid$ (a) Optical photo of the Scenedesmus sp. cell, (b) schematic of the polarized microalgae inside an alternating current electric field. The frequency measurements are expected to deviate in magnitude and phase as the lipid content changes.

due to the induced charging effect (Fig. 1(b) $)^{14-15}$. The propagated RF fields are altered in magnitude and phase depending on the microalgae lipid contents and cells concentration. This is because as the signal frequency increases, the charge distribution in the microalgae is modulated, which in turn can create a polarizing effect, thus enables the lipid quantifications. At a constant concentration of cells, the polarization depends on the compositions and size. The microalgae could be presented as an electrical dipole, which has two pair of electrical charges of equal magnitude but opposite sign, separated by some distance.

The process of developing a correlation for lipid content as function of scattering parameter $S_{11}$ for a specific kind of microalgae, with predetermined cells concentration, starts with taking a sample from a bioreactor, diluting it to a constant cells concentration and then placing it in a RF resonator structure. A network analyzer is used to measure the scattering parameter of the suspended material which is recorded and then processed to extract the change in frequency and magnitude. The actual lipid contents of the microalgae were determined using Soxhlet extraction technique with $n$-hexane ${ }^{16}$ and calibrated against their respective RF signature. The $n$-hexane can extract the non-polar lipids only, which are the interesting lipids for biodiesel production. Hence, the measurements were limited to them only, and the composition is shown in Table 1.

Details of the processing steps:. Sample preparation. Samples were collected at regular intervals and diluted in growth medium to obtain a $4 \mathrm{ml}$ of cell suspension. Figure 2(a) shows the bioreactor from which the samples were collected over days of starvation. The Nile Red stock solution was prepared as described by Siaut et $\mathrm{al}^{17}$. Figure 2(b) shows a comparison between the actual lipid content and the fluorescence intensity determined by the plate reader. The primary stress applied was nitrogen deficiency, where accumulations of more than $70 \%$ (dry basis) have been achieved. This is mainly due to the lack of nitrogen required for protein synthesis, and the excess carbon from photosynthesis is then diverted into lipid production pathway. Figure 2(c) shows the fluorescence microscopy images of cells stained with Nile Red that shows the lipid accumulation with days of nitrogen starvation.

\begin{tabular}{|lc|}
\hline Table $1 \mid$ Scenedesmus sp. microalgae lipid composition \\
Fatty acid methyl ester & \% Composition \\
\hline Methyl myristate & 12.5 \\
Methyl sterate & 6.3 \\
Cis-9-oleic Methyl ester & 12.5 \\
Trans -9-elaidic Methyl ester & 43.8 \\
Methyl linoleate & 18.8 \\
Methyl behanate & 6.3 \\
\hline
\end{tabular}

$R F$ Characterization. In order to confirm the concept, a sample of Scenedesmus sp. microalgal suspension was withdrawn from the bioreactor and concentrated at fixed cells concentration of $1.5 \times$ $10^{6} \mathrm{cells} / \mathrm{ml}$, and then inserted inside the open-ended coaxial resonator as shown in Fig. 3(a). The setup was calibrated before each measurement has been carried out. This calibration ensured that the measurement actually represented the sample under test while excluding the effect of losses and phase shifts due to the cables and host structure which could add noise to the measured signal. A blank was prepared by harvesting the microalgae cells and its lipid content was completely extracted, and then rediluted in growth medium in the same concentration of $1.5 \times 10^{6}$ cells $/ \mathrm{ml}$.

The set of $S_{11}$ parameter measured curves versus frequency for different lipid contents are shown in Fig. 3(b). The RF response of the suspended microalgae pronounces the lipid accumulations as the magnitude of the $S_{11}$ changes gradually with the increase in lipid content. The $S_{11}$ curve shows a smooth performance over frequency and exhibits resonance with specific resonance frequency, bandwidth and magnitude. All of these parameters vary with the lipid content. The resonance frequency of samples free of lipids (i.e., all lipids have been extracted) was found to be higher than that of samples of higher lipid content, due to the change in the effective suspended solution dielectric constant.

Lipid quantification. The lipid content was calibrated against the change in resonance parameters. The resonance can be predicted with the Gaussian distribution function. Gaussian function parameters were correlated with the RF measured parameters, namely, its bandwidth, magnitude and center frequency. A general Gaussian function could be expressed by Eq (1):

$$
G(f)=y_{0}+A e^{-0.5\left(\frac{f-f_{0}}{w}\right)^{2}}
$$

Where, $f, y_{0}, A, w$ and $f_{0}$ are frequency, offset, magnitude, bandwidth and center frequency of the resonance respectively. These set of parameters were correlated against lipid content. The predicted and measured $S_{11}$ response corresponding to lipid contents of $12.10,44.5$ and $73.2 \%$ were drawn against each other as shown in Fig. 3(c). The prediction was carried out from $10 \mathrm{GHz}$ to $13.5 \mathrm{GHz}$, the resonance window. Figure 3 (a) reveals a good agreement between the predictions and measurements, with a standard deviation of \pm 0.001 . The set of parameters $\left\{y_{0}, A, w\right.$ and $\left.f_{0}\right\}$ were determined for different lipid contents by fitting each measured curve in Figure 3(b) with Eq (1). The predicted coefficients are presented in Table 2, with each row represents specific lipid content.

The resonance parameters over lipid content variation are depicted in Fig. 4, which shows that the predicted set of parameters versus lipid content exhibited a linear relationship. As the lipid content increased, the off-set and magnitude parameters changed in the 
(a)

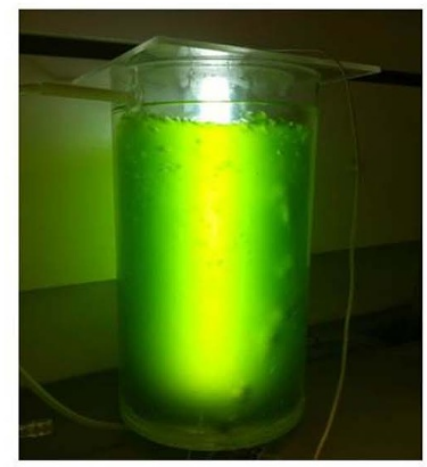

(c)

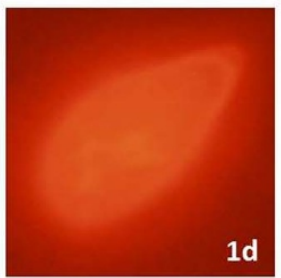

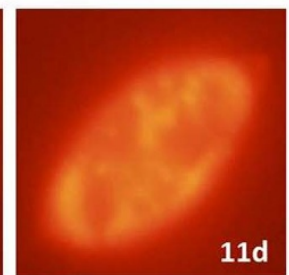

11d (b)

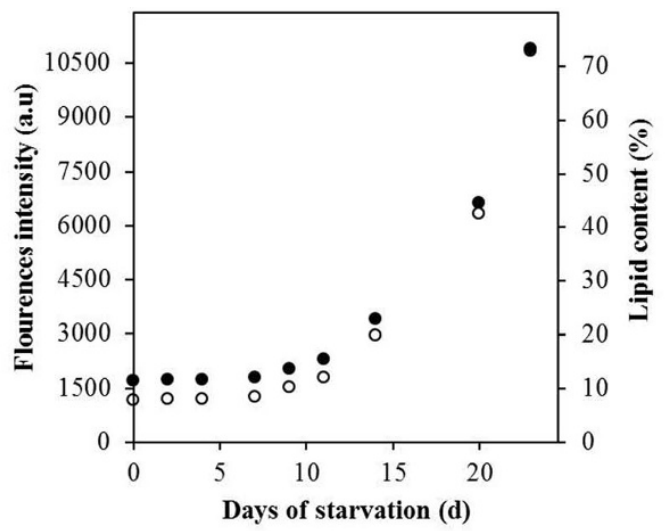

Figure 2 Conventional lipid determination: (a) bioreactor with outer diameter of $10 \mathrm{~cm}$, an inner diameter of $5 \mathrm{~cm}$ and a height of $40 \mathrm{~cm}$. (b) comparison between $(\bigcirc)$ lipid content and $(\bullet)$ relative fluorescence intensity. (c) Fluorescence microscopy images of Scenedesmus sp. cells stained with Nile Red and showing lipid accumulation after 1, 11, 14, 20 and 23 days of nitrogen starvation in cultures.

same manner, while both center frequency and bandwidth exhibited identical behaviour.

The model was validated by measuring RF response of a sample of unknown lipid content. The measured RF signal was fitted to Eq (1) and the associated coefficients were determined and located on the calibration curves shown in Fig. 4. The lipid content that correspond to these values was then determined to be $12.05 \%$. The actual lipid content of the sample was measured using the conventional soxhelt technique and found to be of $12.08 \%$, which is almost identical to that predicted by the model with an error of only $0.2 \%$. This clearly proves that the proposed technique can be used predict accurately the lipid content.

The accuracy of the proposed model is approximately $98 \%$, and the recorded error associated with the lower and higher contents did not exceed $2 \%$ in the entire range of measured lipid content. The error can be further optimized by involving more measured points and by having micro cavity resonator dedicated design. The calibration curve can now be used to determine the lipid content of an unknown sample from measuring the $S_{11}$ frequency response and Gaussian prediction.

\section{Discussion}

New methods for lipid detection and quantification have been place since 1993 and are still getting further attention. However, few methods managed to scale with the volume or contents. While there is tremendous expansion in high algae growth and various environmental conditions, numerous researchers are still only providing a qualitative analysis of the lipids. Moreover, the time of quantitative analysis for these lipids ranges from few hours to days. A method of lipid quantification with less than a minute response detection time is necessary in controlling and rapidly adjusting the growing conditions to achieve a desired objective of the growth.

The RF propagation is confined and guided inside the coaxial cable; the open-end of this cable forms a capacitance within which the microalgae suspension acts as its dielectric material. The initial capacitance corresponds to the lipid free content; exhibits its minimum value with low resistive losses (high quality factor). As the lipid content increases the effective dielectric constant of the solution increases. Figure 5 shows the extracted capacitance and resistance values versus the lipid content. Both capacitance and resistance showed a linear relationship versus the lipid content. The change in the magnitude level of $S_{11}$ parameter is associated with the change in the DC resistance of the suspended solution, whereas the change in the frequency shift is associated with the change in capacitance. Such a strategy streamlined the interpretation of our results in terms of specific RF signatures for these different lipids contents, which could then be attributed solely to the specific nature of respective microalgae particles intrinsic properties.

The selectivity and the sensitivity are two important issues that should be addressed in any detection method. The conventional method, used for lipid determination, involves solvent extraction that requires separation of the solvent from crude extract after the extraction, which is followed by lipid quantification which may require fatty acid composition using chromatography techniques $^{18-19}$. Although these methods have been used widely and have a high accuracy in lipid detection, they are time- and labor-intensive, making it difficult to screen large numbers of algae. To ensure the ability of the detection selectivity of the proposed approach, the frequency responses of other strains of microalgae with identical concentration have been measured and depicted in Fig. 5(b). Figure 5(b) reveals that each microalgae strain was associated with a certain shift in resonance frequency and magnitude; i.e. unique RF signature. Figure 5(b) shows the RF measurements for two different types of microalgae (Scenedesmus sp. And Nannochlorpsis), revealing that each strain has its own resonance frequency, which proves that the proposed method can be used not only to determine the lipid content of other strains, but can also be used to identify the strain. The two strains used are different in size, composition, i.e. different dielectric constants and resistive losses. The extracted lipid shows a higher dielectric constant and better matching as shown in Fig. 5(b-iii).

On the other hand, the sensitivity of the method has been checked by diluting a known concentration of Scenedesmus sp. cells with cultivation medium solution, and the results are shown in Fig. 3(c). 
(a)

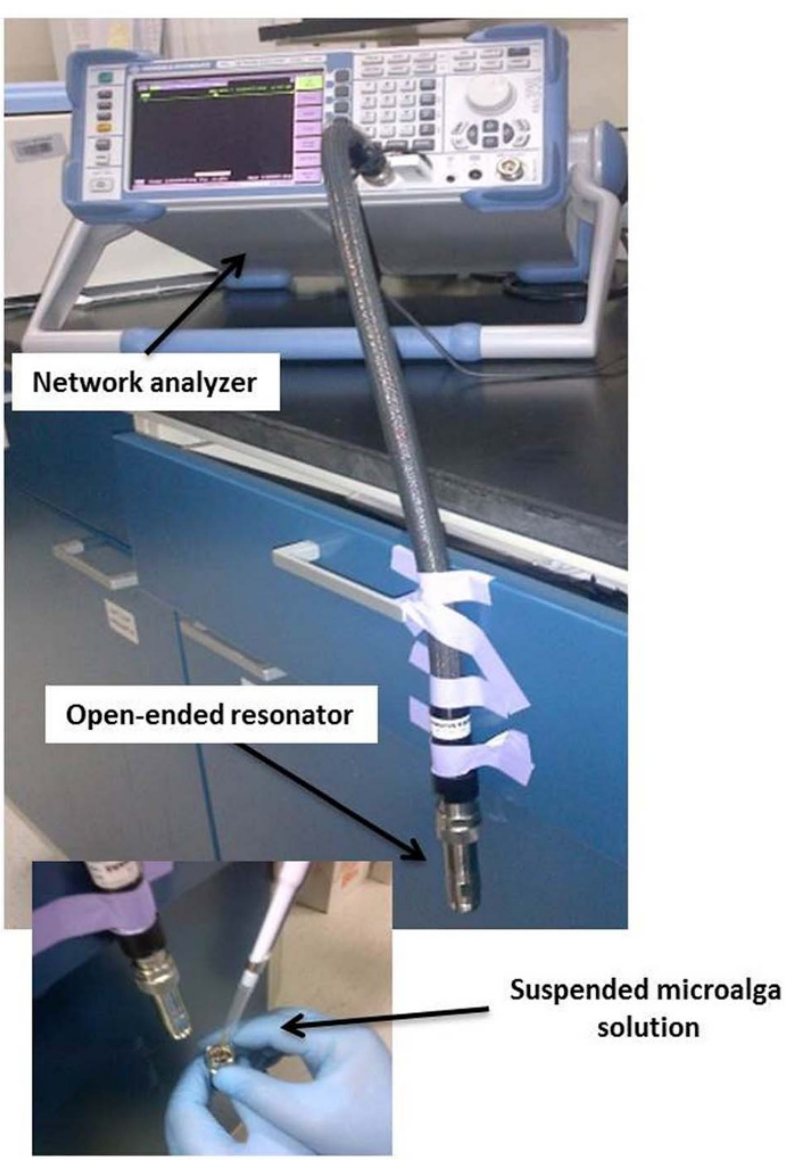

(b)

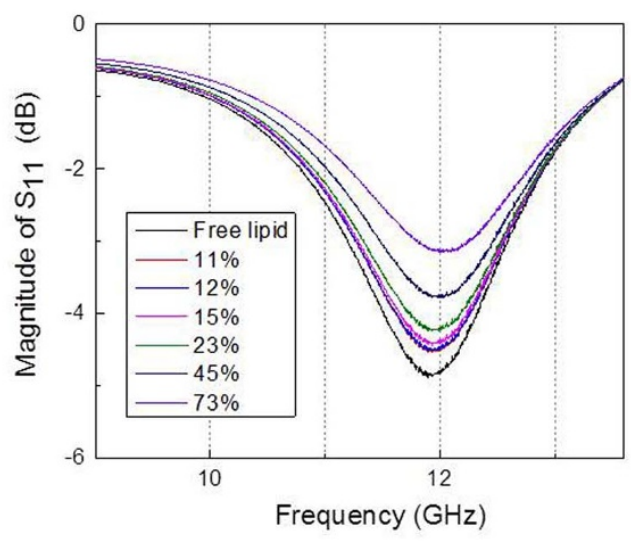

(c)

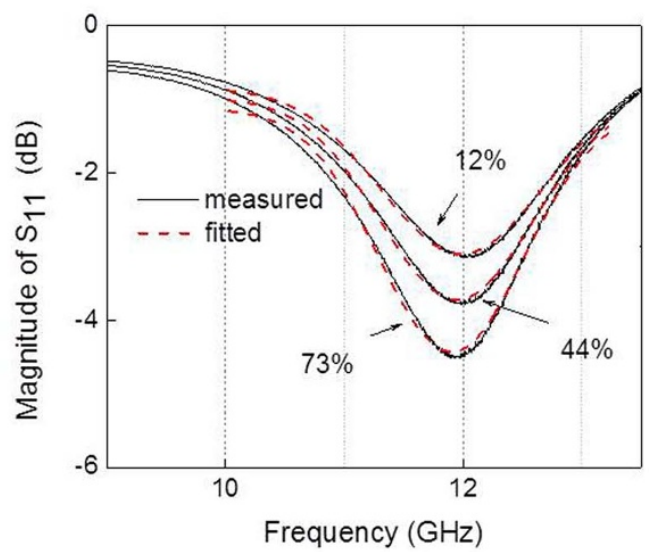

Figure $3 \mid$ Measurements setup and results: (a) Radio frequency measurements setup, (b) $S_{11}$ parameter measurements of suspensions of microalgae with different lipid contents and (c) prediction superimposed with measurement. The samples were withdrawn from the photobioreactor shown in Fig. 2(a) after different starvation durations. The cells of known lipids content were diluted to a constant cells concentration and then loaded into the hosting RF coaxial resonator structure.

This figure shows the sensitivity of the measurements, which reveals that the presented technique is sufficiently sensitive to allow the determination of compounds generated during early-stage cell growth. The figure shows that the frequency shift decreases as microalgae concentration decreases. The RF screening has a higher selectivity than other conventional methods, and thus allows a better limit of detection without the need of any treatment or labeling.

This work is a basic experimental attempt to demonstrate and explore the use of RF method for lipid content determination. The conducted measurements and determined calibration model are applicable to the specific microalgae strain, grown in the specific cultivation medium under certain set of conditions mentioned in this work. If the same strain used is grown in the same medium, under same conditions, then the effects of any possible relative

\begin{tabular}{|lccccc|}
\hline \multicolumn{7}{|l}{ Table 2} & \multicolumn{2}{l}{ prediction coefficients } \\
days & Lipid (\%) & $y_{0}$ & $x_{c}$ & $w$ & $A$ \\
\hline 0 & 0 & -1.034 & 11.896 & 0.593 & -3.848 \\
2 & 11.74 & -0.974 & 11.897 & 0.643 & -3.461 \\
11 & 15.50 & -0.962 & 11.901 & 0.648 & -3.370 \\
14 & 22.93 & -0.938 & 11.911 & 0.654 & -3.219 \\
20 & 44.52 & -0.871 & 11.939 & 0.668 & -2.843 \\
23 & 73.27 & -0.775 & 11.978 & 0.696 & -2.326 \\
\hline
\end{tabular}

variations are eliminated. The same method can be applied to other strains, grown in other media/conditions. To determine the effect of all variables affecting the composition of any strain, a complex experimental design is required. To confirm that this approach can be applied on other strains, a selectivity study has been carried out, as shown in Figure 5 (b).

The proposed method provided a better combination of high sensitivity, quick response, of less than a minute, low cost, high throughput, and ease of use. When compared with other lipid determination techniques, the developed approach was found to be the faster than Raman spectroscopy technique, which needs 2 hours, and cheaper than NMR. The repeatability studies demonstrate a very good accuracy and good predictions. In addition, the proposed technique could be upgraded to be applied in-situ, which will not only pave the way for direct and rapid determination of microalgae lipid content, but also enable the optimization and selection of species and growth conditions. Although in this study, this approach was focused on the determination of the microalgal lipids content, the analytical capability and quantitation algorithms could be applicable to other organisms.

\section{Methods}

Materials. $n$-Hexane, acetone, Nile Red (9-diethylamino-5-benzo $[\alpha]$ phenoxazinone), dimethyl sulfoxide (DMSO), were purchased from Sigma- Aldrich Inc. 


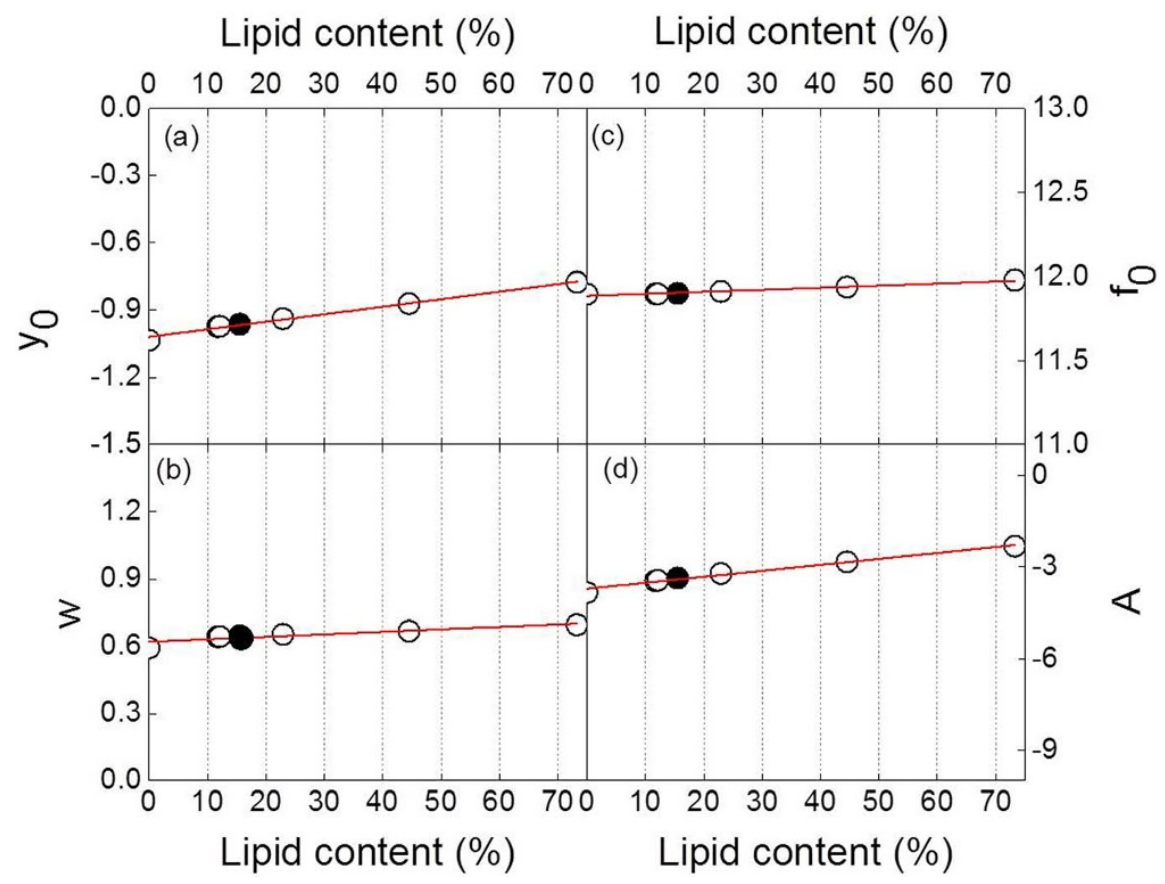

Figure 4 Extracted parameters versus lipid content: (a) the offset, (b) the bandwidth, (c) the center frequency and (d) the resonance magnitude. The measured values used for fitting are depicted by $(\bigcirc)$ and the measured point depicted by $(\bullet)$ is used for validation.

Strain and culture medium. Scenedesmus sp. culture was obtained from Algal Oil Company, Philippines, and cultivated in a nitrogen deficient modified Bassel medium (-N-BBM), which is composed of (in mM); $0.17 \mathrm{CaCl}_{2} \cdot 2 \mathrm{H}_{2} \mathrm{O}, 0.3 \mathrm{MgSO}_{4} \cdot 7 \mathrm{H}_{2} \mathrm{O}, 1.29$ $\mathrm{KH}_{2} \mathrm{PO}_{4}, 0.43 \mathrm{~K}_{2} \mathrm{HPO}_{4}, 0.43 \mathrm{NaCl}, 1 \mathrm{ml} . \mathrm{L}^{-1}$ of Vitamine $\mathrm{B}_{12}$, and $6 \mathrm{ml} . \mathrm{L}^{-1}$ of P-IV solution that consisted of $2 \mathrm{Na}_{2} \mathrm{EDTA} \cdot 2 \mathrm{H}_{2} \mathrm{O}, 0.36 \mathrm{FeCl}_{3} \cdot 6 \mathrm{H}_{2} \mathrm{O}, 0.21 \mathrm{MnCl}_{2} \cdot 4 \mathrm{H}_{2} \mathrm{O}$, $0.37 \mathrm{ZnCl}_{2}, 0.0084 \mathrm{CoCl}_{2} \cdot 6 \mathrm{H}_{2} \mathrm{O}$ and $0.017 \mathrm{Na}_{2} \mathrm{MoO}_{4} \cdot 2 \mathrm{H}_{2} \mathrm{O}$.

Microalgae cultivation. The microalgae was grown in a nitrogen deficient medium (-N-BBM) for three weeks, to enhance the biomass productivity, in a $5 \mathrm{~L}$ bubble

(a)

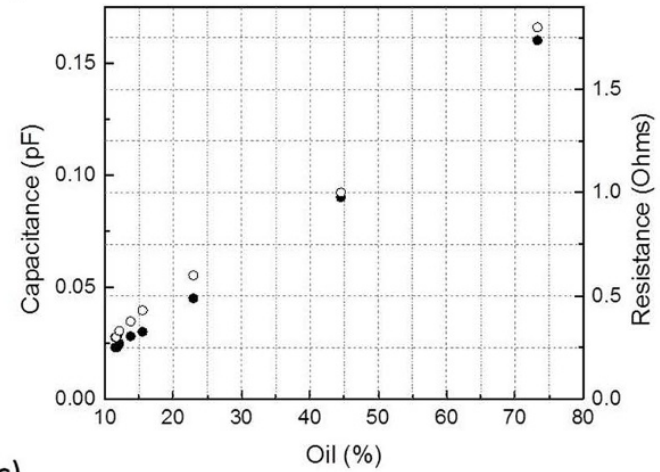

(c)

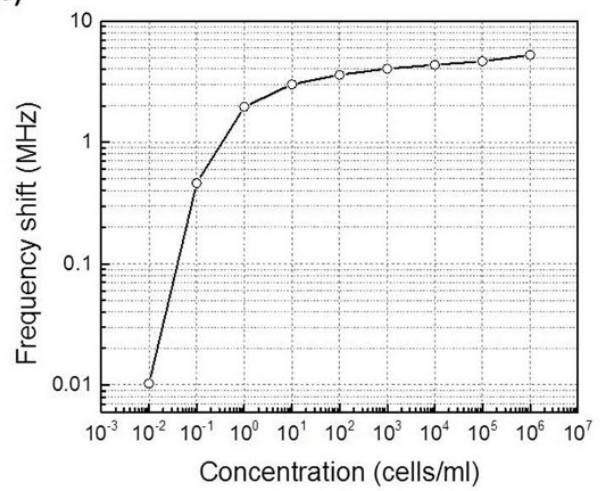

column photobioreactor with an internal illumination. All cultivations in this work were autotrophic, with $\mathrm{CO}_{2}$ naturally present in air bubbled through the system being the sole carbon source.

Prepared medium was sterilized in an autoclave (Hirayama HV-50, Japan) at $121^{\circ} \mathrm{C}$ for $15 \mathrm{~min}$ and cooled to room temperature prior to use. The photobioreactor was illuminated with one $50 \mathrm{~cm}, 60$ watts, white fluorescent light at a light intensity of $120 \mu \mathrm{mol} . \mathrm{m}^{-2} . \mathrm{s}^{-1}$, measured using a light meter under $12 \mathrm{~h}$ light/dark photoperiod automatically controlled by 24 hrs timer (S2402, China). The photobioreactor had an outer diameter of $10 \mathrm{~cm}$, an inner diameter of $5 \mathrm{~cm}$ and a height of $40 \mathrm{~cm}$.

(b)

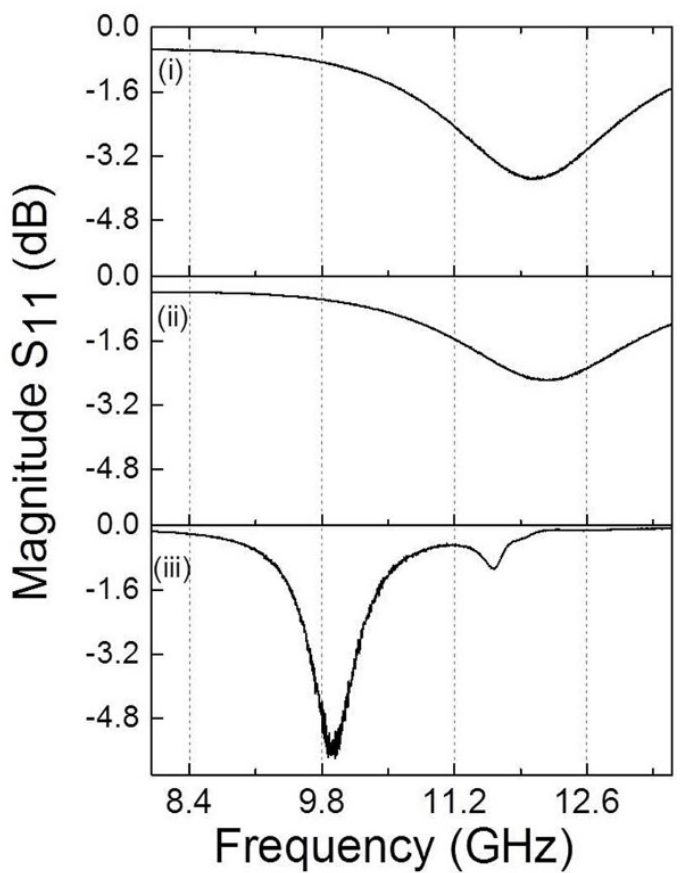

Figure $5 \mid$ (a) Extracted capacitance $(\bullet)$ and resistance $(\bigcirc)$ versus lipid content ratio. (b) selectivity measurements for different microalgae strains (i) Scenedesmus sp. and (ii) Nannochlorpsis and (iii) the extracted lipid only. (c) Sensitivity measurements. 
The cell growth was monitored daily by measuring the optical density at $680 \mathrm{~nm}$ using a spectrophotometer (Shimadzu UV-1800 UV, Kyoto, Japan). The samples were measured twice and the average values were considered. The cells concentration $\left(\right.$ cells. $\mathrm{ml}^{-1}$ ) at any given cultivation time was calculated from a pre-prepared calibration curve of the optical density at $680 \mathrm{~nm}$ versus cells concentration determined using Neubauer Hemocytometer, placed on a microscope (Eclipse LV100 Pol, Nikon, Japan). The dry weight of algal biomass was also determined by filtering the algal suspension using a Whatman filter paper, dried overnight at $105^{\circ} \mathrm{C}$ in an oven (Memmert, Germany) until constant weight.

Calibration curve procedure. Cells concentration was determined by the cell counting technique, where $0.1 \mathrm{~mm}$ deep hemocytometer (PZO, Polandcountry). Different culture dilutions were prepared to calibrate cells concentration versus adsorption using UV spectrophotometer (Hach DR-5000, Germany) at $680 \mathrm{~nm}$. Two $\mathrm{ml}$ of the well mixed culture withdrawn from the flask and diluted to $4 \mathrm{ml}$ in growth medium. This dilution step was repeated four times. $0.1 \mathrm{ml}$ droplet of $1: 16$ diluted were placed on a slide of $0.0025 \mathrm{~m}^{2}$, using Pasteur pipette, and covered with $22 \times$ $22 \mathrm{~mm}$ square cover slip. Cells were allowed to settle for $2 \mathrm{~min}$ and then the number of cells was counted in the four squares at the corner of the hemocytometer grid of nine large squares of $1 \mathrm{~mm}^{2}$ surface area. Total cells concentration per $\mathrm{ml}$ was calculated using Eq (2), from which the initial concentration was determined by multiplying the concentration by 16 .

$$
\text { Cells concentration }=\frac{\text { Total counted cells }}{4} \times 10^{4}
$$

A calibration curve between cell count and the spectrophotometer absorption reading was generated and used in subsequent analysis to convert the spectrophotometer readings into cells concentration. The cells counting and dry weight analysis are standard procedures to determine cells and biomass consecrations. Further details of the procedure can be found elsewhere ${ }^{20,21}$

Lipid content determination. Samples were collected at regular intervals and diluted in growth medium to obtain a $4 \mathrm{ml}$ of cell suspension. The accumulations were monitored by staining the constant concentration samples $\left(1.5 \times 10^{6}\right.$ cells.ml $\left.^{-1}\right)$ with Nile Red that emits a yellow fluorescent signal in the presence of the lipid, and the fluorescents were visualized using fluorescence microscope (Olympus). The Nile Red stock solution was prepared as described by Siaut et $\mathrm{al}^{17}$ by dissolving $0.1 \mathrm{mg}$ of Nile Red in $1 \mathrm{ml}$ acetone, and the solution was stored in the dark at $4^{\circ} \mathrm{C}$. Culture samples $(500 \mu \mathrm{l})$ were placed in an eppendorf tube, span in a centrifuge (Sigma 113, Germany) for $30 \mathrm{~s}$ at $4,000 \mathrm{rpm}$ and $410 \mu \mathrm{l}$ of the supernatant were taken. DMSO $(10 \mu \mathrm{l})$ was then added to promote the accessibility of Nile Red into the cells. The culture was then vortexed and $1 \mu \mathrm{l}$ of Nile Red solution was added followed by $20 \mathrm{~min}$ incubation in the dark. The lipid accumulations were then quantified using Multi-label Plate Reader (Perkin-Elmer, Boston) with black 96-well plates. The cells were harvested by centrifugation and then lyophilized in a freeze drier (Telstar, Terrassa, Spain) operated at $-54^{\circ} \mathrm{C}$ and $0.02 \mathrm{mbar}$ for $6 \mathrm{hrs}$. The actual lipid content was then determined using $\mathrm{n}$-hexane in Soxhlet system.

Radio frequency measurements. The network analyzer (NA) from Rohde and Schwartz (R\&S®ZVL) is commonly used to measure S-parameters, because reflection and transmission of electrical networks are easily measured at high frequencies. The NA involves radio frequency generators that produce power signals at different frequencies, thus when these power signals propagate inside the cultivation medium they scatter back towards the source and some portion of this signal power is absorbed by the microalgae particles. The system was calibrated using the SLOT transmission line techniques for the network analyzer. A typical calibration moves the measurement reference planes to the end of the test cables. Therefore, it excludes the effect of losses and phase shifts that could add noise to the measured signal. Each microalgae suspension, of different lipid content, was exposed to a radio frequency signal with a power of $10 \mathrm{dBm}$ and with a sweep from $8 \mathrm{GHz}$ up to $13.6 \mathrm{GHz}$ (the equipment measurements range capability). The self-resonance frequency of the coaxial cables was ensured to be pushed above $30 \mathrm{GHz}$; so it does not affect the measurements in the mentioned range.

1. Chisti, Y. Biodiesel from microalgae. J. Biotech. Adv. 25, 294-306 (2007)

2. Brennan, L. \& Owende, P. Biofuels from microalgae-a review of technologies for production, processing, and extractions of biofuels and co-products. J. Renewable and Sus. Energy Rev. 14, 557-577 (2010).

3. Stephens, E. et al. An economic and technical evaluation of microalgal biofuels. Nat. biotech. 28, 126-128 (2010).

4. Huo, Y. X. et al. Conversion of proteins into biofuels by engineering nitrogen flux. Nat. biotech. 29, 346-351 (2011).
5. Scragg, A. H., Illman, A. M., Carden, A. \& Shales, S. W. Growth of microalgae with increased calorific values in a tubular bioreactor. Biomass and Bioenergy. 23, 67-73 (2002).

6. Rodolfi, L. et al. Microalgae for oil: Strain selection, induction of lipid synthesis and outdoor mass cultivation in a low-cost photobioreactor. Biotechn. and Bioeng 102, 100-112 (2009).

7. Bligh, E. G. \& Dyer, W. J. A rapid method of total lipid extraction and purification. Can. J. of Biochem. and Phys. 37, 911-917 (1959).

8. Folch, J., Lees, M. \& Sloane Stanley, G. H. A simple method for the isolation and purification of total lipids from animal tissues. J. of Biological Chem. 226, 497-509 (1957).

9. Hara, A. \& Radin, N. S. Lipid extraction of tissues with a low-toxicity solvent Anal. Biochem. 90, 420-426 (1978).

10. Kou, Z., Bei, S., Sun, J. \& Pan, J. Fluorescent measurement of lipid content in the model organism Chlamydomonas reinhardtii. J. of App. Phyco. 25, 1633-1641 (2013).

11. Huawen, W. et al. In vivo lipidomics using single-cell Raman spectroscopy. PNAS 1, 1-6 (2011)

12. Mirsa, D. et al. Noninvasive electrical charcetrization of materials at microwave frequencies using an open-ended coaxial line: test of an improved calibration technique. IEEE MTTS. 38, 8-14 (1990).

13. Schwan, H. P. \& Foster, K. R. RF-Field interactions with biological systems: electrical properties and biophysical mechanisms. IEEE Proc. 68, 104-113 (1980).

14. Grahl, T. \& Märkl, H. Killing of microorganisms by pulsed electric fields. App. Microbio. and Biotech. 45, 148-157 (1999).

15. Sahagún, E. \& Sáenz, J. J. Dielectric nanoparticles: Polarisability reveals identity. Nat. Mat. 11, 748-749 (2012).

16. Taher, H. et al. Supercritical carbon dioxide extraction of microalgae lipid: process optimization and laboratory scale-up. J. of Super Flu. 86, 57-66 (2014).

17. Siaut, M. et al. Oil accumulation in the model green alga Chlamydomonas reinhardtii: characterization, variability between common laboratory strains and relationship with starch reserves. BMC Biotechn. 11, 7 (2011).

18. Eltgroth, M. L., Watwood, R. L. \& Wolfe, G. V. Production and cellular localization of neutral long-chain lipids in the haptophyte algae Isochrysis galbana and Emiliania huxleyi. J. Phyc. 41, 1000-1009 (2005).

19. Chen, W. et al. A high throughput Nile red method for quantitative measurement of neutral lipids in microalgae. J. of microbio. meth. 77, 41-47 (2009).

20. Strober, W. Monitoring cell growth, Current Protocols in Immunology. [21:A.3A.1-A.3A.2.] (John Wiley \& Sons, New York, 2001).

21. Rodolfi, L. et al. Microalgae for oil: Strain selection, induction of lipid synthesis and outdoor mass cultivation in a low-cost photobioreactor. Biotech. and Bioen. 102, 100-112 (2009).

\section{Acknowledgments}

The authors wish to acknowledge the support received from the office of Deputy of Vice Chancellor for Research and Graduate Studies at UAE University under internal university fund code $31 \mathrm{~N} 163$

\section{Author contributions}

M.A. conceived the concept and performed the RF experiments. H.T. and S.A. carried out the cultivation experiments and determined the lipid content using the conventional extraction techniques. M.A., H.T., A.N. and S.A. prepared the manuscript. M.A. supervised the project. All authors discussed the results and commented on the manuscript.

\section{Additional information}

Competing financial interests: The authors declare no competing financial interests.

Reprints and permissions information is available at www.nature.com/reprints.

How to cite this article: Ahmad, M.A., Al-Zuhair, S., Taher, H. \& Hilal-Alnaqbi, A. RF Microalgal lipid content characterization. Sci. Rep. 4, 5108; DOI:10.1038/srep05108 (2014)

This work is licensed under a Creative Commons Attribution-NonCommercialNoDerivs 3.0 Unported License. The images in this article are included in the article's Creative Commons license, unless indicated otherwise in the image credit; if the image is not included under the Creative Commons license, users will need to obtain permission from the license holder in order to reproduce the image. To view a copy of this license, visit http://creativecommons.org/licenses/by-nc-nd/3.0/ 